Copyright (C) 2013 IEEE. Personal use of this material is permitted. Permission from IEEE must be obtained for all other uses, in any current or future media, including reprinting/republishing this material for advertising or promotional purposes, creating new collective works, for resale or redistribution to servers or lists, or reuse of any copyrighted component of this work in other works. 


\title{
Non-Regenerative Multi-Way Relaying: Combining the Gains of Network Coding and Joint Processing
}

\author{
Holger Degenhardt, Student Member, IEEE, Yue Rong, Senior Member, IEEE, and Anja Klein, Member, IEEE
}

\begin{abstract}
In this paper, we consider a non-regenerative multigroup multi-way relaying scenario in which each group consists of multiple half-duplex nodes. Each node wants to share its data with all other nodes within its group. The transmissions are performed via an intermediate non-regenerative half-duplex multi-antenna relay station, termed $\mathrm{RS}$, which spatially separates the different groups. In our proposal, all nodes simultaneously transmit to RS during a common multiple access phase and RS retransmits linearly processed versions of the received signals back to the nodes during multiple broadcast (BC) phases. We propose a novel transmit strategy which exploits analog network coding (ANC) and efficiently combines spatial transceive processing at RS with joint receive processing at each node over multiple BC phases. A closed-form solution for an ANC aware relay transceive filter is introduced and closed-form solutions for the joint receive processing filters at the nodes are presented. Furthermore, self-interference cancellation and successive interference cancellation are exploited at the nodes to improve the joint receive processing. By numerical results, it is shown that the proposed transmit strategy significantly outperforms existing multi-way strategies.
\end{abstract}

Index Terms-Multi-way relaying, non-regenerative relay, analog network coding (ANC), minimum mean-squared error (MMSE).

\section{INTRODUCTION}

Relaying techniques are highly beneficial in wireless communication systems to overcome shadowing effects, to increase the communication range, to improve the energy efficiency and to increase the achievable throughput [1]. Considering applications such as video conferences or multiplayer gaming as well as emergency or sensor applications, usually the data exchange between multiple nodes which belong to a specific group is required. We consider the scenario that each node of a group wants to share its data with all other nodes within its group via an intermediate relay station. For these multi-group multi-way (MGMW) relaying applications, the relay station has to retransmit the received messages such that each node can decode the messages of all other nodes within its group [2]. We focus on non-regenerative relaying schemes to perform these retransmissions, i.e., the transfer function of

The work of Holger Degenhardt is funded by the Deutsche Forschungsgemeinschaft (DFG) under Grant No. K1907/2-2 and is supported by the LOEWE Priority Program Cocoon and by the Graduate School of Computational Engineering at Technische Universität Darmstadt. The work of Yue Rong was supported in part by the Australian Research Councils Discovery Projects funding scheme (project number DP110100736).

H. Degenhardt and A. Klein are with the Communications Engineering Lab, Technische Universität Darmstadt, 64283 Darmstadt, Germany, e-mail: \{h.degenhardt, a.klein\}@nt.tu-darmstadt.de.

Y. Rong is with the Department of Electrical and Computer Engineering, Curtin University, Bentley, WA 6102, Australia, e-mail: y.rong@curtin.edu.au. the relay is equivalent to a memoryless weighting matrix that transforms the (baseband) waveform received at the relay to the (baseband) waveform transmitted from the relay [3].

Single-pair [4]-[13] and multi-pair [14]-[20] relaying scenarios are special cases of MGMW relaying where each group consists only of $N=2$ nodes. For bidirectional communications, the two-way relaying protocol was proposed in [7] to overcome the duplexing loss of conventional one-way relaying schemes [4]-[6]. The filter design for non-regenerative twoway relaying, which enables bidirectional communications between two half-duplex single-antenna or multi-antenna nodes via an intermediate half-duplex multi-antenna relay station, has been investigated in [8]-[13] and references therein. In [14]-[20], the two-way relaying protocol has been extended to consider multiple pairs which simultaneously exchange information via an intermediate non-regenerative multi-antenna relay station. Different relay transceive filter designs have been proposed to exploit self-interference cancellation for bidirectional pairwise communications of single-antenna nodes in [14]-[18]. The authors of [19], [20] have proposed filter designs to enable bidirectional pairwise communications of multi-antenna nodes.

The more general MGMW relaying scenario with $N \geq 2$ nodes per group has been introduced in [2], [21]. In [21], the authors have focused on single-group multi-way relaying with half-duplex nodes and a half-duplex relay station. In [1], [2], the full-duplex multi-group multi-way relay channel has been investigated and time division multiple access (TDMA) has been applied to separate the communications of different groups. Further works on MGMW relaying include [22]-[29]. In [22], [23], non-regenerative multi-way relaying via a halfduplex multi-antenna relay station has been considered for a single group scenario. In [23], different transmit strategies have been investigated and in [22], random beamforming at the relay station has been assumed and the desired signals have been recovered by exploiting the temporal processing capabilities at the nodes. In [24], a non-regenerative MGMW relaying scenario has been investigated and different transmit strategies and relay transceive filter designs have been proposed to spatially separate different groups and to enable the multi-way communications within each group. Different schemes and approaches for regenerative MGMW relaying have been considered in [25]-[29].

In this paper, we focus on non-regenerative MGMW relaying and we consider half-duplex single-antenna nodes and an intermediate half-duplex multi-antenna relay station, termed RS. To exchange the messages within each group of $N$ nodes, all nodes simultaneously transmit in one multiple access 
(MAC) phase to RS and RS retransmits different linearly processed versions of the received signals back to the nodes in the subsequent $N-1$ broadcast (BC) phases. Thus, $N$ time slots are required to perform the exchange of all messages which is equal to the required number of time slots for performing the data exchange by direct communications between the nodes without using a relay station. Up to now, either the optimization of the spatial processing at RS [23], [24] or of the temporal processing at the nodes [22] has been investigated because an efficient combination of both approaches is not straightforward. To enable an efficient combination, network coding [30] can be exploited and due to focusing on nonregenerative relaying, we propose to exploit analog network coding (ANC) [31].

We propose a new transmit strategy and a novel relay transceive filter design to perform the retransmissions at RS during the $\mathrm{BC}$ phases. The proposed transmit strategy exploits ANC and efficiently combines the spatial processing at RS with joint temporal receive processing at the nodes. By ANC, we mean that instead of spatially separating the received signals at RS as considered in [23], [24], we propose to retransmit different linear combinations of the received signals in each $\mathrm{BC}$ phase. Afterwards, the individual signals are recovered at the nodes by exploiting the temporal processing capabilities and by subtracting self-interferences. To increase the temporal processing gain at the nodes, we propose to specifically change the linear combinations of the retransmitted signals at RS to reduce the linear dependencies. Furthermore, by the novel relay transceive filter design, we take into account that no power should be wasted at RS neither for retransmitting interfering signals nor for suppressing interferences which can be canceled at the nodes.

In detail, we propose that the spatial processing at RS is based on considering a unicast (UC) signal per group which is desired at one node and a multicast (MC) signal per group which is desired at the remaining nodes of the corresponding group in each BC phase similar to the hybrid uni-/multicasting transmit strategy of [24]. However, in contrast to [24], the UC and the MC signal of each group are spatially superimposed due to exploiting ANC. Thus, we do not waste power at RS for spatially separating these signals because these signals can be separated by utilizing the temporal processing capabilities at the nodes. Furthermore, to increase the temporal processing gain at the nodes whilst utilizing the spatial processing capabilities at RS efficiently, we propose that the retransmission of one signal per group is suppressed in each BC phase. Additionally, to increase the receive power of the desired signals, the power which is wasted at RS for retransmitting interfering signals and for suppressing interferences which can be canceled at the nodes is minimized by the proposed relay transceive filter. Similar to [22], we apply minimum meansquared error (MMSE) based receive filters to perform joint receive processing at the nodes over all $\mathrm{BC}$ phases and we perform successive interference cancellation (SIC). However, in contrast to [22], we do not apply random beamforming at RS. We propose an approach to efficiently design the relay transceive filter and to exploit the spatial processing capabilities at RS. The selection of the UC, the MC and the suppressed signal in each BC phase influences the achievable data rates. Thus, we propose a low-complexity approach to perform a proper selection.

The rest of the paper is organized as follows. In Section II, the system model for MGMW relaying is presented. In Section III, the proposed transmit strategy is introduced. The proposed ANC aware relay transceive filter design is presented in Section IV. Joint temporal processing at the nodes and the corresponding MMSE-SIC filter design are presented in Section V. A low-complexity approach to perform the selection of the UC, the MC and the suppressed signals is presented in Section VI. For simplicity of the notations, a single multi-way group is considered in Sections III-VI and the extension to consider multiple multi-way groups is presented in Section VII. Numerical results in Section VIII confirm the analytical investigations and Section IX concludes the paper.

Notations: The operators $\operatorname{tr}(\cdot), \operatorname{diag}[\cdot], \otimes$ denote the sum of the main diagonal elements of a matrix, the construction of a diagonal matrix with the elements contained in the vector and the Kronecker product of matrices, respectively. The operator $\Re[\cdot]$ denotes the real part of a scalar and $E[\cdot]$ denotes the expectation over the random variables within the brackets. The operators $|\cdot|,\|\cdot\|_{2},\|\cdot\|_{F}$ denote the norm of a complex number, the Euclidean norm of a complex vector and the Frobenius norm of a complex matrix, respectively. The vectorization operator $\operatorname{vec}(\mathbf{Z})$ stacks the columns of matrix $\mathbf{Z}$ into a vector. The operator $\operatorname{vec}_{M, N}^{-1}(\cdot)$ is the revision of the operator $\operatorname{vec}(\cdot)$, i.e., a vector of length $M N$ is sequentially divided into $N$ smaller vectors of length $M$ which are combined to a matrix with $M$ rows and $N$ columns. Furthermore, $\mathbf{I}_{M}$ denotes an identity matrix of size $M$.

\section{System MOdeL}

In this paper, a multi-group multi-way relaying scenario as shown in Fig. 1 is considered. The scenario consists of multiple single-antenna nodes and a multi-antenna relay station RS which is equipped with $L$ antennas. Each node has to exchange a message with all other nodes within its group. These multi-way communications are performed via a single subcarrier and, in general, $G \geq 1$ groups and $N \geq 2$ nodes per group are considered. Thus, the total number of nodes is given by $K=G \cdot N$.

The variable $\mathrm{S}_{k}, k=1,2, \ldots, K$, is used to label the nodes. The maximum transmit power at each node and at the relay station RS is $P_{\mathrm{MS}}$ and $P_{\mathrm{RS}}$, respectively. All transmitted signals are assumed to be statistically independent and the noises at RS and at the nodes are assumed to be independent and identically distributed (i.i.d.) additive white Gaussian noise (AWGN) with zero mean and variances $\sigma_{\mathrm{n}, \mathrm{RS}}^{2}$ and $\sigma_{\mathrm{n}}^{2}$, respectively.

The system equations for the proposed scheme are presented in the equivalent baseband. The transmit signal of $\mathrm{S}_{k}$ is given by $s_{k} \in \mathbb{C}^{1 \times 1}$ with $\mathrm{E}\left[s_{k} s_{k}^{\mathrm{H}}\right]=P_{\mathrm{MS}}$ and $N$ time slots are required to perform the information exchange between all nodes within the same group. The channel $\mathbf{h}_{k} \in \mathbb{C}^{L \times 1}$ from node $\mathrm{S}_{k}$ to $\mathrm{RS}$ is assumed to be constant during these $N$ time slots and channel reciprocity is assumed. Such quasistatic channel model has been widely used in two-way and 


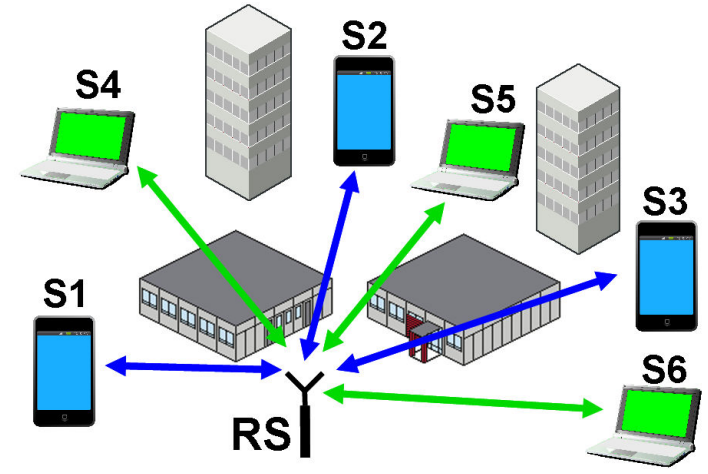

Fig. 1. Multi-group multi-way relaying scenario with $G=2$ groups and $N=3$ nodes per group. The multi-antenna relay station RS enables both groups to simultaneously exchange information.

in multi-way relay communications [8], [9], [11]-[13], [15], [16], [22]-[24].

In the first time slot $t=1$, all nodes simultaneously transmit to RS and the superposition of these transmit signals is received at RS. We refer to this time slot as MAC phase and it is a common assumption in multi-way relaying [22][24] that the received signals at RS are synchronized. Thus, the received signal at RS can be written as

$$
\mathbf{y}_{\mathrm{RS}}=\sum_{k=1}^{K} \mathbf{h}_{k} s_{k}+\mathbf{n}_{\mathrm{RS}},
$$

where $\mathbf{n}_{\mathrm{RS}} \in \mathbb{C}^{L \times 1}$ represents the complex white Gaussian noise vector at $\mathrm{RS}$.

In the $N-1$ subsequent time slots, RS retransmits different linearly processed versions of the superimposed received signals back to the nodes and we refer to these time slots as $\mathrm{BC}$ phases. In time slots $t=2,3, \ldots, N$, the received signal $\mathbf{y}_{\mathrm{RS}}$ is linearly processed at RS using the transceive filter matrices $\mathbf{G}_{t} \in \mathbb{C}^{L \times L}$ which are derived in Section IV. Thus, the received signal $y_{k, t} \in \mathbb{C}^{1 \times 1}$ using the receive filter coefficient $d_{k, t} \in \mathbb{C}^{1 \times 1}$ at node $\mathrm{S}_{k}$ in time slot $t$ is given by

$$
y_{k, t}=d_{k, t}\left(\mathbf{h}_{k}^{\mathrm{T}} \mathbf{G}_{t} \mathbf{y}_{\mathrm{RS}}+n_{k, t}\right),
$$

where $n_{k, t} \in \mathbb{C}^{1 \times 1}$ represents the complex white Gaussian noise at $\mathrm{S}_{k}$ in the $t^{\text {th }}$ time slot.

In the remainder of this section and in Sections III-VI, we focus on a single group scenario, i.e., $G=1$, to simplify the notations. The extension to a multi-group scenario is presented in Section VII.

In the following, the system equations are extended to enable the consideration of joint receive processing with SIC at the nodes. Let us define a vector $\mathbf{a}_{k, l} \in \mathbb{C}^{(N-1) \times 1}$ to describe the channel coefficients for the transmission from $\mathrm{S}_{l}$ to $\mathrm{S}_{k}$ in the $N-1$ BC phases as

$$
\mathbf{a}_{k, l}=\left(d_{k, 2} \mathbf{h}_{k}^{\mathrm{T}} \mathbf{G}_{2} \mathbf{h}_{l}, d_{k, 3} \mathbf{h}_{k}^{\mathrm{T}} \mathbf{G}_{3} \mathbf{h}_{l}, \ldots, d_{k, N} \mathbf{h}_{k}^{\mathrm{T}} \mathbf{G}_{N} \mathbf{h}_{l}\right)^{\mathrm{T}} .
$$

Furthermore, let $\mathbf{W}_{k} \in \mathbb{C}^{(N-1) \times(N-1)}$ be a matrix to perform joint linear receive processing at $\mathrm{S}_{k}$ and $\mathbf{n}_{\mathrm{ov}, k} \in \mathbb{C}^{(N-1) \times 1}$ be the overall noise vector for reception at $\mathrm{S}_{k}$ given by

$$
\mathbf{n}_{\mathrm{ov}, k}=\left(d_{k, 2} n_{\mathrm{ov}, k, 2}, d_{k, 3} n_{\mathrm{ov}, k, 3}, \ldots, d_{k, N} n_{\mathrm{ov}, k, N}\right)^{\mathrm{T}}
$$

with $n_{\mathrm{ov}, k, t}=\mathbf{h}_{k}^{\mathrm{T}} \mathbf{G}_{t} \mathbf{n}_{\mathrm{RS}}+n_{k, t}$. Now, we can write the received signals at $S_{k}$ after joint linear processing over the $N-1$ BC phases as

$\mathbf{y}_{k}=\mathbf{W}_{k}\left(\mathbf{a}_{k, 1}, \mathbf{a}_{k, 2}, \ldots, \mathbf{a}_{k, N}\right) \cdot\left(s_{1}, s_{2}, \ldots, s_{N}\right)^{\mathrm{T}}+\mathbf{W}_{k} \mathbf{n}_{\mathrm{ov}, k}$.

Remark: To decouple the temporal and the spatial processing for the relay transceive filter design in Section IV, the receive filter coefficients $d_{k, t}$ and the receive processing matrix $\mathbf{W}_{k}$ are designed separately. In particular, $\mathbf{W}_{k}$ depends on the relay transceive filter design, while the receive filter coefficients $d_{k, t}$ do not, as shown later.

To compute the relay transceive filter and to perform selfinterference cancellation and SIC, channel state information (CSI) is required at RS and at the nodes. The required CSI can be obtained through channel training and estimation [32]-[36]. In this paper, it is assumed that RS has perfect knowledge of the channel coefficients in $\mathbf{h}_{k}$ to compute the relay transceive filter. Furthermore, it is assumed that each node $S_{k}$ perfectly cancels the back-propagated self-interferences $\mathbf{y}_{\mathrm{SI}, k}=\mathbf{a}_{k, k} s_{k}$. To perfectly cancel the back-propagated self-interferences $\mathbf{y}_{\mathrm{SI}, k}$, it is assumed that each node has perfect knowledge of the corresponding channel coefficients $g_{k, k, t}=\mathbf{h}_{k}^{\mathrm{T}} \mathbf{G}_{t} \mathbf{h}_{k}$, $t=2,3, \ldots, N$ describing the overall channels. Additionally, it is assumed that the nodes can perform perfect SIC to reduce the interferences through stream-wise decoding. To perform SIC, the channel coefficients $g_{k, l, t}=\mathbf{h}_{k}^{\mathrm{T}} \mathbf{G}_{t} \mathbf{h}_{l}, \forall l \neq k$, are assumed to be perfectly known at each node and perfect cancellation is assumed.

To estimate the transmit signal of $\mathrm{S}_{l}$ at $\mathrm{S}_{k}$, the $l^{\text {th }}$ row vector $\mathbf{w}_{k, l}$ of $\mathbf{W}_{k}$ is used which corresponds to the joint receive processing vector for estimating this signal. The computation of the vector $\mathbf{w}_{k, l}$ is presented in Section V. To consider SIC, let $\mathcal{N}_{k, l}$ be a subset which contains the indices of the nodes whose transmit signals are already decoded at $\mathrm{S}_{k}$ and let this subset include the index $k$ to consider perfect self-interference cancellation. Now, the expected signal, interference and noise powers when estimating the transmit signal of $S_{l}$ at $S_{k}$ can be written as

$$
\begin{aligned}
P_{\mathrm{S}, k, l} & =P_{\mathrm{MS}}\left|\mathbf{w}_{k, l} \mathbf{a}_{k, l}\right|^{2}, \\
P_{\mathrm{I}, k, l} & =P_{\mathrm{MS}} \sum_{j=1, j \notin \mathcal{N}_{k, l}}^{N}\left|\mathbf{w}_{k, l} \mathbf{a}_{k, j}\right|^{2}, \\
P_{\mathrm{N}, k, l} & =\mathrm{E}\left[\mathbf{w}_{k, l} \mathbf{n}_{\mathrm{ov}, k} \mathbf{n}_{\mathrm{ov}, k}^{\mathrm{H}} \mathbf{w}_{k, l}^{\mathrm{H}}\right],
\end{aligned}
$$

respectively. Thus, for decoding the first transmit signal, all other transmit signals are considered as interferences and for decoding the last transmit signal, all these interferences are canceled in advance.

For performance comparison, the maximum achievable sum rate of multi-way relaying, cf. [23], [24], is considered and the corresponding equations are presented in the following. Assuming that Gaussian codebooks are used for each signal, the maximum achievable data rate from $S_{l}$ to $S_{k}$ is given by

$$
C_{k, l}=\frac{1}{N} \log _{2}\left(1+P_{\mathrm{S}, k, l}\left(P_{\mathrm{I}, k, l}+P_{\mathrm{N}, k, l}\right)^{-1}\right),
$$


where $N$ is the number of required time slots to perform all multi-way transmissions. The maximum achievable multi-way rate for the transmit signal of $\mathrm{S}_{l}$ is determined by the minimum over all achievable rates from $S_{l}$ to any other node within the same group. Thus, it is given by

$$
C_{l, \max }=(N-1) \cdot \min _{\forall k \in \mathcal{N}_{l}, k \neq l} C_{k, l},
$$

where $\mathcal{N}_{l}$ contains the indices of all nodes within the group of $\mathrm{S}_{l}$. The achievable sum rate of the multi-way relay system is given by

$$
C_{\text {sum }}=\sum_{l=1}^{K} C_{l, \max }
$$

\section{SUPERIMPOSED UNI-/MULTICASTING TRANSMIT STRATEGY}

In this section, we propose a superimposed uni-/multicasting transmit strategy, termed S-UC/MC, which is designed to perform all transmissions in one MAC and $N-1$ BC phases. Accordingly, only $N$ time slots are required to perform the exchange of all messages which is equal to the required number of time slots for performing the data exchange by direct communications between the nodes without using a relay station. The proposed S-UC/MC transmit strategy exploits ANC and efficiently combines the spatial processing capabilities at RS and the temporal processing capabilities at the nodes.

To exchange all messages in $N-1 \mathrm{BC}$ phases, different linear combinations of the transmitted signals have to be received at each node in each BC phase. The proposed S-UC/MC transmit strategy is based on retransmitting spatially processed linear combinations of all received signals such that the spatial processing capabilities at RS are utilized efficiently and the temporal processing capabilities at the nodes can be exploited. Using $\mathbf{y}_{\mathrm{RS}}$ of (1), the retransmitted linear combination in time slot $t$ is given by $\mathbf{G}_{t} \mathbf{y}_{\mathrm{RS}}$ and to describe the proposed spatial processing at RS, we consider four different signal categories which are introduced in the following.

First, we consider desired signals at the nodes in each BC phase similar to the transmit strategies presented in [23], [24]. We propose that the transmit signal $s_{m_{t}}$, termed MC signal, is desired at all nodes $\mathrm{S}_{k}, k \neq m_{t}$, where $m_{t}$ is the index of the MC signal in time slot $t$. This MC signal changes in each $\mathrm{BC}$ phase and the indices of the different $\mathrm{MC}$ signals are contained in the vector $\mathbf{m}=\left(m_{2}, \ldots, m_{N}\right)$. Additionally, we propose that the transmit signal $s_{u}$, termed UC signal, is desired at node $\mathrm{S}_{m_{t}}, u \neq m_{t} \forall t=2, \ldots, N$, where $u$ is the index of the unicast signal. The UC signal is the same in all $\mathrm{BC}$ phases. To summarize, the desired signal at node $\mathrm{S}_{k}$ in time slot $t$ is given by $s_{l}$ with

$$
l=\left\{\begin{array}{ccc}
m_{t} & \text { if } \quad k \neq m_{t}, \\
u & \text { if } \quad k=m_{t} .
\end{array}\right.
$$

Due to changing the $\mathrm{MC}$ signal in each $\mathrm{BC}$ phase, every transmit signal $s_{l}, l \neq k$, is desired at $\mathrm{S}_{k}$ in one of the $\mathrm{BC}$ phases. Using this approach, we can focus the relay transmit power on as few signals as possible because only one MC and one UC signal are desired at all nodes in each BC phase. For the retransmission of the desired signals, we propose to exploit ANC. By ANC, we mean that instead of spatially separating both desired signals as considered in [23], [24], we propose to spatially superimpose the desired UC and MC signals in each $\mathrm{BC}$ phase and to recover the individual signals by utilizing the temporal processing capabilities at the nodes.

Secondly, a suppressed signal is considered in each BC phase which has to be spatially separated from the desired signals. The consideration of a suppressed signal increases the temporal processing gain at the nodes in case of $N>2$ because it reduces the linear dependencies between the retransmitted signals of the different BC phases. Suppressing more than one signal or all remaining signals as considered in [23], [24] is not beneficial because it would reduce the temporal processing gain and would require more antennas at RS for spatially separating desired and suppressed signals. Thus, we propose that only one signal $s_{o_{t}}$ is suppressed in each BC phase, where $o_{t}$ is the index of the suppressed signal in time slot $t$. The suppressed signal changes in each time slot and the indices of the suppressed signals are contained in the vector $\mathbf{o}=\left(o_{2}, \ldots, o_{N}\right), u \neq o_{t} \neq m_{t} \forall t=2, \ldots, N$. For suppressing the signal $s_{o_{t}}$, we propose an MMSE based separation which considers the noise at RS as described in detail in Section IV.

Thirdly, self-interference signals (SISs) are considered. For the spatial processing at RS, $s_{k}$ is considered as SIS at node $\mathrm{S}_{k}$ in time slot $t$ if $k \neq o_{t}$. SISs can be canceled at the nodes before performing temporal receive processing and SIC. Thus, no power should be wasted at RS neither for retransmitting SISs nor for spatially separating SISs from the desired signals. Based on this, we propose to consider SISs with respect to the power constraint at RS. Thus, spatially processed linear combinations of the desired signals and the SISs are retransmitted by RS in each BC phase whereas these signals are spatially separated in [23], [24].

Fourthly, the remaining signals (RMSs) at the nodes are considered. The signal $s_{l}$ is considered as RMS at node $\mathrm{S}_{k}$ in time slot $t$ if

$$
\begin{aligned}
\{l\} \cap\left\{m_{t}, k, o_{t}\right\}=\emptyset & \text { for } k \neq m_{t}, \\
\{l\} \cap\left\{u, k, o_{t}\right\}=\emptyset & \text { for } k=m_{t} .
\end{aligned}
$$

Due to considering joint temporal receive processing over all $\mathrm{BC}$ phases at the nodes, the RMSs can be used to improve the overall performance because each RMS in one BC phase is a desired signal in another $\mathrm{BC}$ phase. Based on this, we propose that RMSs are treated in the same way as SISs for the spatial processing at RS. However, RMSs are not known at the nodes and thus, cannot be canceled before performing temporal receive processing. Therefore, the interferences caused by RMSs have to be reduced or canceled at the nodes when estimating a desired signal by performing temporal receive processing and SIC. The proposed approach exploits ANC and instead of spatially separating the RMSs from the desired signals as considered in [23], [24], spatially processed linear combinations of these signals are retransmitted by RS in each $\mathrm{BC}$ phase.

Considering the introduced signal categories, we propose that the spatial processing at RS should minimize the mean- 
squared error (MSE) for the transmission of the desired signals in time slot $t$ given by

$$
\begin{aligned}
& \min _{\mathbf{G}_{t}} \mathrm{E}\left[\sum_{l=1}^{K}\left|s_{k}-\hat{s}_{k, l}\right|^{2}\right], k=\left\{\begin{array}{ccc}
m_{t} & \text { if } & l \neq m_{t}, \\
u & \text { if } & l=m_{t},
\end{array}\right. \\
& \text { s.t. } \sum_{k=1}^{K}\left\|\mathbf{G}_{t} \mathbf{h}_{k}\right\|_{2}^{2} P_{\mathrm{MS}}+\left\|\mathbf{G}_{t}\right\|_{\mathrm{F}}^{2} \sigma_{\mathrm{n}, \mathrm{RS}}^{2} \leq P_{\mathrm{RS}},
\end{aligned}
$$

where $\hat{s}_{k, l}$ is the estimate of $s_{k}$ at node $\mathrm{S}_{l}$ assuming that the SISs and the RMSs can be perfectly canceled at the nodes due to exploiting the temporal processing capabilities. Thus, $\hat{s}_{k, l}$ only contains the desired signal, the suppressed signal and noise and it is given by

$$
\hat{s}_{k, l}=d_{l, t} \mathbf{h}_{l}^{\mathrm{T}} \mathbf{G}_{t}\left(\mathbf{h}_{k} s_{k}+\mathbf{h}_{o_{t}} s_{o_{t}}+\mathbf{n}_{\mathrm{RS}}\right)+d_{l, t} n_{l, t} .
$$

An intuitive explanation of the proposed spatial processing at $\mathrm{RS}$ with respect to the introduced signal categories can be given as follows. Considering (14a), the MSE for the transmission of the desired signals is minimized. In the estimate of each desired signal (15), the impact of the suppressed signal $s_{o_{t}}$ is considered, and thus, the retransmission of $s_{O_{t}}$ is suppressed at RS to minimize (14a). The SISs and the RMSs are not considered in the estimate of each desired signal (15) because it is assumed that these signals can be suppressed or canceled by utilizing the temporal processing capabilities at the nodes. However, the SISs and the RMSs are considered in the power constraint at RS (14b). Thus, no power is wasted at RS with respect to minimizing the MSE (14a) neither for retransmitting SISs and RMSs nor for spatially separating SISs and RMSs from the desired signals.

An exemplary overview of the proposed S-UC/MC transmit strategy is given in Table I for a scenario consisting of $N=4$ nodes. At node $\mathrm{S}_{k}$, the signal $s_{k}$ is self-interference. Thus, it is not shown in Table I because it can be perfectly canceled before performing temporal receive processing. In this example, the UC signal $s_{1}$ is desired at node $\mathrm{S}_{t}$ in time slot $t$ which is marked by $u$ in the table. Furthermore, the MC signal $s_{m_{t}}$ is desired at the remaining nodes in each time slot which is marked by $m$ in the table. Additionally, the signal $s_{O_{t}}$ is considered as suppressed signal at each node in time slot $t$. The suppressed signal is marked by $o$ in the table. The RMSs which are only considered with respect to the power constraint at $\mathrm{RS}$ in each $\mathrm{BC}$ phase are marked by $*$. The individual signals are recovered at the nodes by performing joint temporal receive processing over the received signals of all $\mathrm{BC}$ phases as described in Section V.

TABLE I

PROPOSED S-UC/MC TRANSMIT STRATEGY FOR A MULTI-WAY GROUP OF $N=4$ NODES, $u=1, \mathbf{m}=(2,3,4), \mathbf{o}=(3,4,2)$.

\begin{tabular}{c|lll|lll|lll|lll} 
& \multicolumn{3}{|c|}{ signals at $\mathrm{S}_{1}$} & \multicolumn{3}{|c|}{ signals at $\mathrm{S}_{2}$} & \multicolumn{3}{|c|}{ signals at $\mathrm{S}_{3}$} & \multicolumn{3}{|c}{ signals at $\mathrm{S}_{4}$} \\
$t$ & $s_{2}$ & $s_{3}$ & $s_{4}$ & $s_{1}$ & $s_{3}$ & $s_{4}$ & $s_{2}$ & $s_{1}$ & $s_{4}$ & $s_{2}$ & $s_{3}$ & $s_{1}$ \\
\hline 2 & $m$ & $o$ & $*$ & $u$ & $o$ & $*$ & $m$ & $*$ & $*$ & $m$ & $o$ & $*$ \\
3 & $*$ & $m$ & $o$ & $*$ & $m$ & $o$ & $*$ & $u$ & $o$ & $*$ & $m$ & $*$ \\
4 & $o$ & $*$ & $m$ & $*$ & $*$ & $m$ & $o$ & $*$ & $m$ & $o$ & $*$ & $u$
\end{tabular}

Spatially superimposing the desired signals $s_{u}$ and $s_{m_{t}}$ and considering that only $s_{O_{t}}$ has to be spatially separated from these desired signals in each $\mathrm{BC}$ phase reduces the required

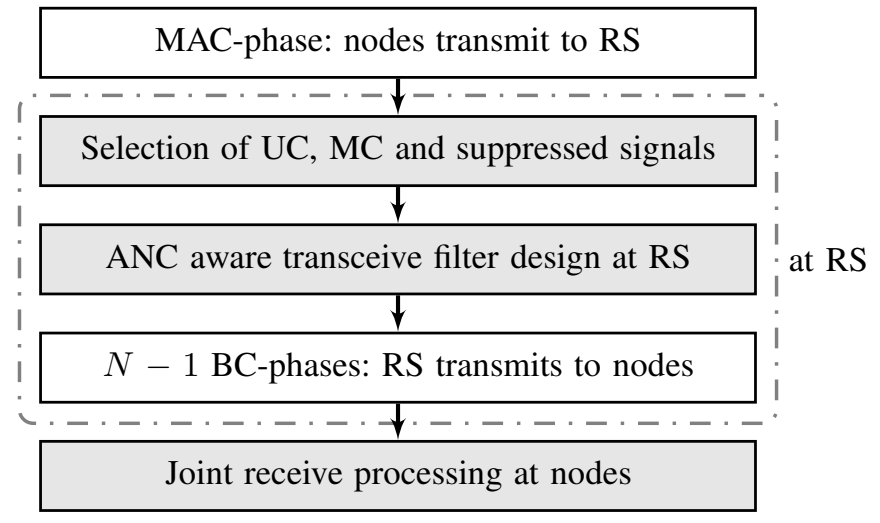

Fig. 2. Overview of one cycle of the proposed S-UC/MC transmit strategy.

number $L$ of antennas at RS compared to approaches which do not exploit ANC and require the spatial separation of all signals. The proposed S-UC/MC transmit strategy only requires one spatial dimension at RS to retransmit the desired signals. In case of $N>2$, one additional spatial dimension is required to spatially separate $s_{o_{t}}$ from the desired signals in each BC phase. Thus, the required number of antennas at RS is given by $L=1$ for $N=2$ and by $L=2$ for $N>2$. If more than the required number of antennas are available at RS, the proposed relay transceive filter of Section IV utilizes these antennas to minimize the MSE (14a).

To provide a general overview, one cycle of the proposed $\mathrm{S}-\mathrm{UC} / \mathrm{MC}$ transmit strategy is illustrated in Fig. 2. In the MAC phase, all nodes simultaneously transmit to RS. Afterwards, the processing at RS is performed in three steps. First, RS performs a selection of the UC, the MC and the suppressed signals for the different BC phases. An approach to perform an efficient selection of these signals is presented in Section VI. Secondly, the ANC aware relay transceive filters for the different $\mathrm{BC}$ phases are computed based on the preselected signals as presented in Section IV. Thirdly, RS retransmits the received signals to the nodes in $N-1$ different BC phases after linearly processing these signals with the corresponding relay transceive filter for each BC phase. Finally, each node performs joint receive processing over the received signals during the $\mathrm{BC}$ phases to estimate all desired signals as described in Section V.

\section{ANC AWARE TRANSCEIVE FILTER DESIGN AT RS}

In this section, we propose an ANC aware relay transceive filter design for the proposed S-UC/MC transmit strategy, cf. Fig. 2. For given receive filters at the nodes, the MMSE problem (14) is convex and an analytical relay transceive filter solution can be derived. However, this requires that the receive filters at the nodes are known in advance. In the following, we propose an approach to compute the receive filters at the nodes independent of the relay transceive filter. Afterwards, we present the derivation for the proposed ANC aware relay transceive filter design considering the received noise powers at the nodes.

For a single antenna node as assumed in this paper, the receive filter is just a single coefficient which rotates and weights 
the receive signal. Considering the proposed S-UC/MC strategy, a MC signal is retransmitted to $N-1$ different nodes in each $\mathrm{BC}$ phase. If the relay transceive filter has to cope with $N-1$ different channel rotations for retransmitting this MC signal, the performance is reduced. Thus, it is important for the nodes which receive the MC signal to reverse these channel rotations and we propose to calculate the receive filter coefficient at each node $\mathrm{S}_{k}$ in time slot $t$ according to $d_{k, t}=\left(\mathbf{h}_{k}^{\mathrm{T}} \mathbf{h}_{l}\right)^{\mathrm{H}} /\left|\mathbf{h}_{k}^{\mathrm{T}} \mathbf{h}_{l}\right|$, where $l=m_{t}$ if $k \neq m_{t}$ and $l=u$ if $k=m_{t}$. This is the matched filter coefficient for the overall channel of the desired signal assuming an identity matrix for the processing at RS. These receive filters decouple the spatial processing at RS and the temporal processing at the nodes.

If we now design the relay transceive filter $\mathbf{G}_{t}$ to minimize (14), the solution for $\mathbf{G}_{t}$ does not consider the noise powers at the nodes. However, the noise powers at the nodes should be considered with respect to the power constraint at RS to increase the achievable data rates. To achieve this, we propose to consider an additional receive coefficient $\alpha_{t}$ at all nodes and to solve the joint optimization problem of $\alpha_{t}$ and $\mathbf{G}_{t}$ as it is considered for multiple-input multiple-output Tx filter design in [37], [38].

Thus, the joint optimization problem for the ANC aware relay transceive filter $\mathbf{G}_{t}$ and the receive coefficient $\alpha_{t}$ with respect to the transmit power constraint at $\mathrm{RS}$ in time slot $t$ is given by

$$
\begin{gathered}
\left\{\alpha_{t}, \mathbf{G}_{t}\right\}=\underset{\alpha_{t}, \mathbf{G}_{t}}{\arg \min } \mathrm{E}\left[\sum_{l=1}^{K}\left|s_{k}-\alpha_{t} \hat{s}_{k, l}\right|^{2}\right], \\
\text { s.t. } \sum_{l=1}^{K}\left\|\mathbf{G}_{t} \mathbf{h}_{l}\right\|_{2}^{2} P_{\mathrm{MS}}+\left\|\mathbf{G}_{t}\right\|_{\mathrm{F}}^{2} \sigma_{\mathrm{n}, \mathrm{RS}}^{2} \leq P_{\mathrm{RS}},
\end{gathered}
$$

where $k=m_{t}$ if $l \neq m_{t}$ or $k=u$ if $l=m_{t}$ is the index of the desired $\mathrm{MC}$ or $\mathrm{UC}$ signal at $\mathrm{S}_{l}$ in time slot $t$, respectively.

Thus, the MSE for the transmission from $S_{k}$ to $S_{l}$ in time slot $t$ is given by

$$
\begin{aligned}
& \mathrm{E}\left[\left|s_{k}-\alpha_{t} \hat{s}_{k, l}\right|^{2}\right] \\
& =P_{M S}-2 \Re\left[\alpha_{t} d_{l, t} \mathbf{h}_{l}^{\mathrm{T}} \mathbf{G}_{t} \mathbf{h}_{k} P_{M S}\right] \\
& +\left|\alpha_{t}\right|^{2}\left|d_{l, t}\right|^{2} \mathbf{h}_{l}^{\mathrm{T}} \mathbf{G}_{t} \mathbf{h}_{k} P_{\mathrm{MS}} \mathbf{h}_{k}^{\mathrm{H}} \mathbf{G}_{t}^{\mathrm{H}} \mathbf{h}_{l}^{*} \\
& +\left|\alpha_{t}\right|^{2}\left|d_{l, t}\right|^{2} \mathbf{h}_{l}^{\mathrm{T}} \mathbf{G}_{t} \mathbf{h}_{o_{t}} P_{\mathrm{MS}} \mathbf{h}_{o_{t}}^{\mathrm{H}} \mathbf{G}_{t}^{\mathrm{H}} \mathbf{h}_{l}^{*} \\
& +\left|\alpha_{t}\right|^{2}\left|d_{l, t}\right|^{2} \sigma_{n, \mathrm{RS}}^{2} \mathbf{h}_{l}^{\mathrm{T}} \mathbf{G}_{t} \mathbf{G}_{t}^{\mathrm{H}} \mathbf{h}_{l}^{*}+\sigma_{n}^{2}\left|\alpha_{t}\right|^{2}\left|d_{l, t}\right|^{2},
\end{aligned}
$$

where $k$ is again the index of the desired signal at $\mathrm{S}_{l}$ in time slot $t$. The objective function (16a) is non-convex since $\mathbf{G}_{t}$ and $\alpha_{t}$ appear jointly in third-order degree or higher. However, $\alpha_{t}$ can be assumed to be positive real-valued and the MSE of (17) as well as the constraint (16b) are convex with respect to $\mathbf{G}_{t}$. Thus, a unique solution for problem (16a) can be obtained by using Lagrangian optimization. Let matrices $\Upsilon^{(k)}$ and $\Upsilon$ be given by

$$
\begin{aligned}
\mathbf{\Upsilon}^{(k)} & =P_{\mathrm{MS}} \mathbf{h}_{k} \mathbf{h}_{k}^{\mathrm{H}}, \\
\mathbf{\Upsilon} & =\sum_{k=1}^{K} P_{\mathrm{MS}} \mathbf{h}_{k} \mathbf{h}_{k}^{\mathrm{H}}+\sigma_{n, \mathrm{RS}}^{2} \mathbf{I}_{L} .
\end{aligned}
$$

Using matrix $\Upsilon$ of (18b) in (16a) and considering the power constraint (16b), the Lagrangian function with the Lagrangian multiplier $\eta$ results in

$$
L\left(\mathbf{G}_{t}, \alpha_{t}, \eta\right)=\sum_{l=1}^{K} F\left(\mathbf{G}_{t}, \alpha_{t}, t, l\right)-\eta\left(\operatorname{tr}\left(\mathbf{G}_{t} \mathbf{\Upsilon} \mathbf{G}_{t}^{\mathrm{H}}\right)-P_{\mathrm{RS}}\right),
$$

where $F\left(\mathbf{G}_{t}, \alpha_{t}, t, l\right)=\mathrm{E}\left[\left|s_{k}-\alpha_{t} \hat{s}_{k, l}\right|^{2}\right]$ of (17). From the Lagrangian function, the Karush-Kuhn-Tucker (KKT) conditions can be derived and $\eta$ can be computed, which is presented in the Appendix. To solve the optimization problem based on the predefined receive filters at the nodes, we define the matrix $\mathbf{K}_{t}$ based on the first KKT condition (33a) as

$$
\begin{aligned}
\mathbf{K}_{t} & =\sum_{l=1}^{K}\left[\boldsymbol{\Upsilon}^{(k)^{\mathrm{T}}} \otimes\left(\mathbf{h}_{l}^{*} \mathbf{h}_{l}^{\mathrm{T}}\right)+\mathbf{\Upsilon}^{\left(o_{t}\right)^{\mathrm{T}}} \otimes\left(\mathbf{h}_{l}^{*} \mathbf{h}_{l}^{\mathrm{T}}\right)\right] \\
& +\sum_{l=1}^{K}\left[\sigma_{n, \mathrm{RS}}^{2} \mathbf{I}_{L} \otimes\left(\mathbf{h}_{l}^{*} \mathbf{h}_{l}^{\mathrm{T}}\right)\right]+\mathbf{\Upsilon}^{\mathrm{T}} \otimes \frac{K \sigma_{\mathrm{n}}^{2}}{P_{\mathrm{RS}}} \mathbf{I}_{L}
\end{aligned}
$$

Taking into account that $\alpha_{t}$ is positive real-valued and using (20), (33) and (39), the ANC aware relay transceive filter which solves problem (16) is given by

$$
\mathbf{G}_{t}=\frac{1}{\alpha_{t}} \cdot \operatorname{vec}_{L, L}^{-1}\left(\mathbf{K}_{t}^{-1} \operatorname{vec}\left(\sum_{l=1}^{K} P_{\mathrm{MS}} \mathbf{h}_{l}^{*} d_{l, t}^{*} \mathbf{h}_{k}^{\mathrm{H}}\right)\right),
$$

where $k$ is again the index of the desired signal at $\mathrm{S}_{l}$ in time slot $t$ and

$$
\alpha_{t}=\sqrt{\frac{\operatorname{tr}\left(\widetilde{\mathbf{G}}_{t} \mathbf{\Upsilon} \widetilde{\mathbf{G}}_{t}^{\mathrm{H}}\right)}{P_{\mathrm{RS}}}}
$$

with the auxiliary matrix $\widetilde{\mathbf{G}}_{t}$ given by

$$
\widetilde{\mathbf{G}}_{t}=\operatorname{vec}_{L, L}^{-1}\left(\mathbf{K}_{t}^{-1} \operatorname{vec}\left(\sum_{l=1}^{K} P_{\mathrm{MS}} \mathbf{h}_{l}^{*} d_{l, t}^{*} \mathbf{h}_{k}^{\mathrm{H}}\right)\right) .
$$

Thus, we have obtained an analytical MMSE solution for the ANC aware relay transceive filter which is optimized regarding our proposed S-UC/MC transmit strategy.

\section{JoInt Receive Processing By ApPlying MMSE-SIC FILTERING AT NODES}

In this section, we present the concept of joint receive processing over the $N-1 \mathrm{BC}$ phases with SIC at the nodes for the proposed S-UC/MC transmit strategy, cf. Fig. 2. In [22], joint receive processing has been investigated for random relay transceive filter design. In the following, we discuss the concept of SIC and introduce an approach to determine a UC/MC decoding order for the proposed S-UC/MC transmit strategy. Afterwards, we present the receive filter design at the nodes for joint receive processing over all $\mathrm{BC}$ phases by utilizing SIC.

\section{A. SIC Decoding Order}

SIC is applied at the nodes, i.e., when decoding the first transmit signal, all other transmit signals are considered as interference and when decoding the last transmit signal, all these interferences are canceled in advance. We assume 
that the decoding order for each node $\mathrm{S}_{k}$ is defined as $\mathbf{q}_{k}=\left(q_{k, 1}, \ldots, q_{k, N-1}\right)$, where $q_{k, 1}$ is the index of the node whose transmit signal is decoded first and $q_{k, N-1}$ is the index of the node whose transmit signal is decoded last. To achieve high sum rates, the optimization of the decoding order is important. In this section, we propose a suboptimal lowcomplexity approach to obtain a UC/MC decoding order for the proposed S-UC/MC transmit strategy.

The average receive power at the nodes of the UC signal is higher than that of any MC signal over all BC phases because the UC signal is never suppressed at RS. Furthermore, the UC signal interferes with every MC signal because it is either considered as desired signal, as SIS or as RMS for the spatial processing at RS. Thus, we propose to decode the UC signal first at the nodes $\mathrm{S}_{k}$, i.e., $q_{k, 1}=u \forall k \neq u$. The different MC signals are received equally strong on average. To define the decoding order for these signals, we consider that the maximum achievable multi-way rate for the transmit signal of $\mathrm{S}_{l}$ is determined by the minimum over all achievable rates from $S_{l}$ to any other node (10). Thus, we propose that the decoding order of the MC signals should be equal at all nodes because the signal to interference ratio increases on average in each decoding step which increases the achievable data rates. To achieve this, the MC signals can be decoded in decreasing order of the respective indices of the transmitting nodes. In summary, the decoding order for nodes $\mathrm{S}_{k}, k \neq u$, is

$$
\mathbf{q}_{k}=(u, i, j, \ldots, l),
$$

where $N \geq i>j>l \geq 1,\{i, j, \ldots, l\} \cap\{k, u\}=\emptyset$ describe the indices of the nodes in decreasing order excluding $k$ and $u$. For node $\mathrm{S}_{u}$, the decoding order is

$$
\mathbf{q}_{u_{g}}=(i, j, \ldots, l)
$$

where $N \geq i>j>l \geq 1,\{i, j, \ldots, l\} \cap\{u\}=\emptyset$.

\section{B. Joint Receive Processing at Nodes}

After defining the decoding order by considering the proposed S-UC/MC transmit strategy, the joint processing matrix $\mathbf{W}_{k}$ of (5) has to be determined for $\mathrm{S}_{k}$. To determine $\mathbf{W}_{k}$, we apply an MMSE based filter design as considered in [22]. For this filter design, perfect self-interference cancellation and perfect SIC are assumed. Considering the decoding order $\mathbf{q}_{k}$ at $\mathrm{S}_{k}$, we can rewrite (5) using (3) as

$$
\begin{aligned}
\mathbf{y}_{k} & =\mathbf{W}_{k}\left(\mathbf{a}_{k, q_{k, N-1}}, \ldots, \mathbf{a}_{k, q_{k, 1}}\right) \cdot\left(s_{q_{k, N-1}}, \ldots, s_{q_{k, 1}}\right)^{\mathrm{T}} \\
& +\mathbf{W}_{k} \mathbf{n}_{\mathrm{ov}, k},
\end{aligned}
$$

where $\mathbf{W}_{k}=\left(\mathbf{w}_{k, q_{k, N-1}}^{\mathrm{T}}, \ldots, \mathbf{w}_{k, q_{k, 1}}^{\mathrm{T}}\right)^{\mathrm{T}}$. To compute $\mathbf{W}_{k}$ based on an MMSE design considering SIC, we introduce the matrix

$$
\mathbf{A}_{k, l}=\left(\mathbf{a}_{k, q_{k, N-1}}, \ldots, \mathbf{a}_{k, q_{k, l}}\right), l=1,2, \ldots, N-1,
$$

with $\mathbf{a}_{k, q_{k, l}}$ of (3) and where $l$ is an index for the decoding step. Using matrix $\mathbf{A}_{k, l}$ of (27), the MMSE filter for jointly estimating the signal transmitted by $\mathrm{S}_{q_{k, l}}$ at $\mathrm{S}_{k}$ over the $N-1$ $\mathrm{BC}$ phases is given by

$$
\mathbf{w}_{k, q_{k, l}}=P_{\mathrm{MS}} \mathbf{a}_{k, q_{k, l}}^{\mathrm{H}}\left(P_{\mathrm{MS}} \mathbf{A}_{k, l} \mathbf{A}_{k, l}^{\mathrm{H}}+\sigma_{n}^{2} \mathbf{I}_{N-1}+\mathbf{N}_{\mathrm{RS}, k}\right)^{-1},
$$

where $\mathbf{N}_{\mathrm{RS}, k}=\operatorname{diag}\left(\left\|\mathbf{h}_{k}^{\mathrm{T}} \mathbf{G}_{2}\right\|_{2}^{2} \sigma_{n, \mathrm{RS}}^{2}, \ldots,\left\|\mathbf{h}_{k}^{\mathrm{T}} \mathbf{G}_{N}\right\|_{2}^{2} \sigma_{n, \mathrm{RS}}^{2}\right)$ and where the interferences of all signals which are decoded in previous decoding steps are assumed to be perfectly canceled by applying SIC.

\section{SElection of UC, MC AND SUPPRESSED SIGNALS}

In this section, we present an approach for the selection of the UC, the MC and the suppressed signals for the proposed S-UC/MC transmit strategy, cf. Fig. 2. The selection of the UC signal $s_{u}$ has an impact on the achievable data rates. Furthermore, the selection of the MC signal $s_{m_{t}}$ with respect to the selection of the signal $s_{O_{t}}$ which has to be suppressed in time slot $t$ influences the achievable MSE of (14) and thus, influences the achievable data rates. Thus, we introduce an approach to determine the index $u$ of the signal which should be unicasted and an approach to obtain a suitable sorting of $\mathbf{m}$ and $\mathbf{o}$ which contain the indices of the signals which should be multicasted and suppressed, respectively.

\section{A. Selection of UC signal}

To determine the UC signal $s_{u}$ which has to be selected to achieve the highest sum rate, an exhaustive search over the signals transmitted by all nodes within the group has to be performed. However, this increases the computational complexity. Thus, we introduce a suboptimal approach which is based on the cross-correlations between the different channels $\mathbf{h}_{k}$ over which the signals $s_{k}$ are transmitted from the nodes to RS. The intention of the suboptimal approach is to select a UC signal which is transmitted over a channel which is highly correlated with all other channels because the UC signal is either considered as desired signal, as SIS or as RMS for the relay transceive filter design. The sum of the cross-correlations between the channel $\mathbf{h}_{k}$ and all other channels is given by

$$
c_{k}=\sum_{l=1}^{N} \frac{\left|\mathbf{h}_{k}^{\mathrm{H}} \mathbf{h}_{l}\right|^{2}}{\mathbf{h}_{k}^{\mathrm{H}} \mathbf{h}_{k} \mathbf{h}_{l}^{\mathrm{H}} \mathbf{h}_{l}} .
$$

Thus, we propose to determine the index $u$ of the UC signal according to $u=\underset{k}{\arg \max } c_{k}, 1 \leq k \leq N$.

\section{B. Selection of $M C$ and suppressed signals}

To achieve a low MSE for the MC signal, cf. (14), the correlation between the channel $\mathbf{h}_{m_{t}}$ over which the MC signal is transmitted from $\mathrm{S}_{m_{t}}$ to $\mathrm{RS}$ and the channel $\mathbf{h}_{o_{t}}$ over which the suppressed signal $s_{o_{t}}$ is transmitted from $\mathrm{S}_{o_{t}}$ to $\mathrm{RS}$ should be as low as possible in each BC phase. Without loss of generality, we propose to keep the sorting of $\mathbf{o}$ fixed and to change the sorting of $\mathbf{m}$ to achieve low correlations. Thus, the optimization of $\mathbf{m}$ can be described as

$$
\mathbf{m}=\underset{\mathbf{m}}{\arg \min } \sum_{t=2}^{N} \frac{\left|\mathbf{h}_{m_{t}}^{\mathrm{H}} \mathbf{h}_{o_{t}}\right|^{2}}{\mathbf{h}_{m_{t}}^{\mathrm{H}} \mathbf{h}_{m_{t}} \mathbf{h}_{o_{t}}^{\mathrm{H}} \mathbf{h}_{o_{t}}} .
$$

To obtain the optimal sorting of $\mathbf{m}$ is a combinatorial problem. Thus, we propose a stepwise low-complexity algorithm as presented in Table II to obtain a suitable sorting of $\mathbf{m}$ for the proposed S-UC/MC transmit strategy. 
TABLE II

Algorithm TO OBTAIN A SUITABLE SORTING OF m

1) Define a set $\mathcal{N}_{\mathrm{MC}}$ which contains all indices of $\mathbf{m}$.

2) For $t=2$ to $t=N$ :

Set $m_{t}=\underset{o_{j}}{\arg \min } \frac{\left|\mathbf{h}_{o_{j}}^{\mathrm{H}} \mathbf{h}_{o_{t}}\right|^{2}}{\mathbf{h}_{o_{j}}^{\mathrm{H}} \mathbf{h}_{o_{j}} \mathbf{h}_{o_{t}}^{\mathrm{H}} \mathbf{h}_{o_{t}}}, o_{j} \in \mathcal{N}_{\mathrm{MC}}$,

$j=2,3, \ldots, N$.

Remove $m_{t}$ from the set $\mathcal{N}_{\text {MC }}$.

3) If $m_{N}=o_{N}$, perform a reallocation for $m_{N}$ :

Search for $a=\underset{m_{j}}{\arg \min } \frac{\left|\mathbf{h}_{m_{j}}^{\mathrm{H}} \mathbf{h}_{o_{N}}\right|^{2}}{\mathbf{h}_{m_{j}}^{\mathrm{H}} \mathbf{h}_{m_{j}} \mathbf{h}_{o_{N}}^{\mathrm{H}} \mathbf{h}_{O_{N}}}, j=2,3, \ldots, N$.

Set $m_{j}=m_{N}$ and $m_{N}=a$.

\section{EXTENSION TO MULTIPLE MULTI-WAY GROUPS}

Up to now, we have focused on single-group multi-way relaying. In this section, we describe how the proposed S-UC/MC transmit strategy can be extended to consider multiple groups.

Considering multiple groups, inter-group interferences have to be considered. We propose to apply the S-UC/MC transmit strategy separately to each group and to suppress inter-group interferences by utilizing the spatial processing capabilities at RS. Thus, the relay transceive filter has to be modified to suppress inter-group interferences. The derivation of the relay transceive filter is similar to the derivation in Section IV and only the solution for $\mathbf{K}_{t}$ changes as follows:

$$
\begin{aligned}
\mathbf{K}_{t} & =\sum_{l=1}^{K}\left[\mathbf{\Upsilon}^{(k)^{\mathrm{T}}} \otimes\left(\mathbf{h}_{l}^{*} \mathbf{h}_{l}^{\mathrm{T}}\right)+\mathbf{\Upsilon}^{\left(o_{g, t}\right)^{\mathrm{T}}} \otimes\left(\mathbf{h}_{l}^{*} \mathbf{h}_{l}^{\mathrm{T}}\right)\right] \\
& +\sum_{l=1}^{K}\left[\sigma_{n, \mathrm{RS}}^{2} \mathbf{I}_{L} \otimes\left(\mathbf{h}_{l}^{*} \mathbf{h}_{l}^{\mathrm{T}}\right)\right]+\mathbf{\Upsilon}^{\mathrm{T}} \otimes \frac{K \sigma_{\mathrm{n}}^{2}}{P_{\mathrm{RS}}} \mathbf{I}_{L} \\
& +\sum_{l=1}^{K} \sum_{j=1, j \neq \mathcal{N}_{k}}^{K}\left[\mathbf{\Upsilon}^{(j)^{\mathrm{T}}} \otimes\left(\mathbf{h}_{l}^{*} \mathbf{h}_{l}^{\mathrm{T}}\right)\right],
\end{aligned}
$$

where $\mathcal{N}_{k}$ contains the indices of all nodes within the group of $\mathrm{S}_{k}$ including the index $k$ itself. Thus, the ANC aware relay transceive filter for multiple groups using $\mathbf{K}_{t}$ of (31) is given by

$$
\mathbf{G}_{t}=\frac{1}{\alpha_{t}} \cdot \operatorname{vec}_{L, L}^{-1}\left(\mathbf{K}_{t}^{-1} \operatorname{vec}\left(\sum_{l=1}^{K} P_{\mathrm{MS}} \mathbf{h}_{l}^{*} d_{l, t}^{*} \mathbf{h}_{k}^{\mathrm{H}}\right)\right),
$$

where $k$ is the index of the desired signal at $\mathrm{S}_{l}$ in time slot $t$ and where $\alpha_{t}$ is defined as given in (22).

The MMSE-SIC filter design of Section V and the selection of the UC and MC signals presented in Section VI are applied separately to each group without considering intergroup interferences. Thus, to extend the proposed S-UC/MC transmit strategy to consider multiple groups, only the relay transceive filter has to be modified as described above.

\section{Simulation RESUlts}

In this section, we investigate the performance of the proposed S-UC/MC transmit strategy for multi-way relaying through numerical simulations. We assume all channels to be i.i.d. Rayleigh fading channels with zero-mean and unit variance and all noises to be i.i.d. complex circularly symmetric Gaussian with zero mean and variance $\sigma_{\mathrm{RS}}^{2}=\sigma_{\mathrm{n}}^{2}$. All simulation results are averaged over 1000 independent channel realizations. In the simulations, we set the maximum transmit power at RS to be equal to the maximum transmit power at each node, i.e., $P_{\mathrm{RS}}=P_{\mathrm{MS}}$. The ratio between the maximum transmit power $P_{\mathrm{MS}}$ at the nodes and the noise level $\sigma_{\mathrm{n}}^{2}$ is termed average receive SNR at RS.

For comparison, the following approaches are considered which have a similar complexity as the proposed S-UC/MC transmit strategy:

- U/MC:ZF [24]: hybrid uni-/multicasting transmit strategy of [24] considering a zero-forcing (ZF) filter at RS,

- U/MC:MMSE [24]: hybrid uni-/multicasting transmit strategy of [24] considering an MMSE filter at RS,

- MMSE-SIC [22]: joint receive processing approach of [22] considering random beamforming at RS,

- U/MC:MMSE-SIC [22+24]: straightforward combination of the hybrid uni-/multicasting transmit strategy of [24] considering an MMSE filter at RS and the joint receive processing approach of [22] at the nodes,

- S-UC/MC:UC/MC-opt.: proposed S-UC/MC transmit strategy with optimal selection of the UC and MC signals obtained by an exhaustive search,

- S-UC/MC:UC/MC-random: proposed S-UC/MC transmit strategy with random selection of the $\mathrm{UC}$ and MC signals.

\section{A. Single-Group Multi-Way Scenario}

Fig. 3 shows the average achievable sum rates versus the number $L$ of antennas at RS for a scenario consisting of $G=1$ group with $N=4$ single antenna nodes. For these simulations, we set $P_{\mathrm{MS}}$ to be $15 \mathrm{~dB}$ above the noise level $\sigma_{\mathrm{n}}^{2}$, i.e. the average receive SNR at RS is $15 \mathrm{~dB}$. For this scenario, the approaches which are based on the proposed S-UC/MC transmit strategy outperform all other approaches because the proposed transmit strategy efficiently combines ANC aware spatial processing at $\mathrm{RS}$ and joint temporal receive processing at the nodes. Considering the proposed transmit strategy, the relative performance gap between an optimal and a random selection of the UC and MC signals is approximately $13 \%$ for $L=3$ and decreases for an increasing number of antennas at RS, e.g., the gap is approximately $9 \%$ for $L=4$ and $7 \%$ for $L=5$. Considering a selection of the UC and MC signals as proposed in Section VI, the proposed S-UC/MC transmit strategy performs in between S-UC/MC:UC/MC-random and S-UC/MC:UC/MC-opt.. To spatially separate the received signals at RS, $L \geq N$ antennas are required. Thus, U/MC:ZF [24] starts from $L=4$ antennas. For U/MC:MMSE [24], a solution can be obtained for all $L$ due to an MMSE based separation of the signals. The performance gain of the proposed S-UC/MC transmit strategy compared to U/MC:MMSE [24] increases for a decreasing number of antennas at RS, e.g., the gain is approximately $23 \%$ for $L=5$ and $63 \%$ for $L=3$ because the proposed S-UC/MC transmit strategy exploits the temporal processing capabilities at the nodes. The performance gain of the proposed S-UC/MC transmit strategy compared to MMSE-SIC [22] increases for an increasing number of antennas at RS, e.g., the gain is approximately $38 \%$ for $L=3$ and $83 \%$ for $L=5$ because the proposed S-UC/MC 


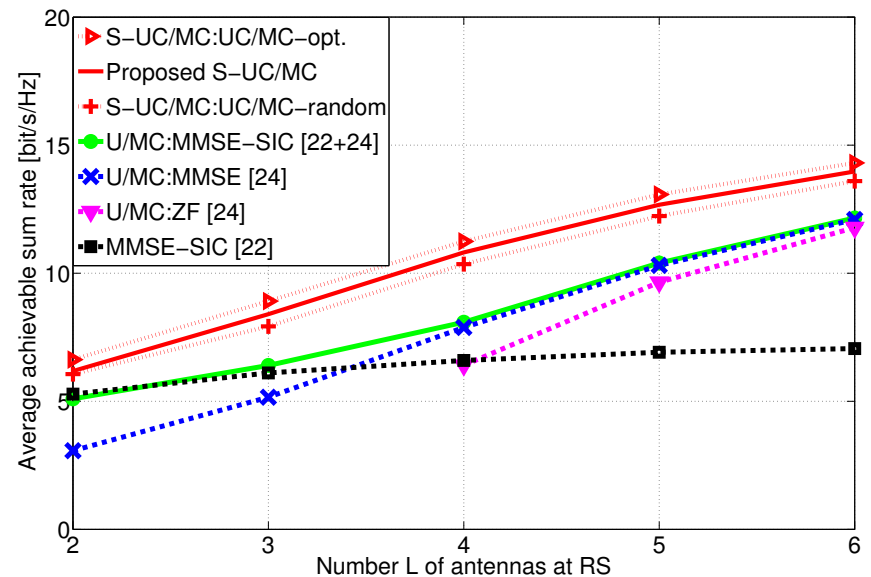

Fig. 3. Average achievable sum rates versus number $L$ of antennas at RS for an average receive $\mathrm{SNR}=15 \mathrm{~dB}$ at $\mathrm{RS}, G=1, N=4$.

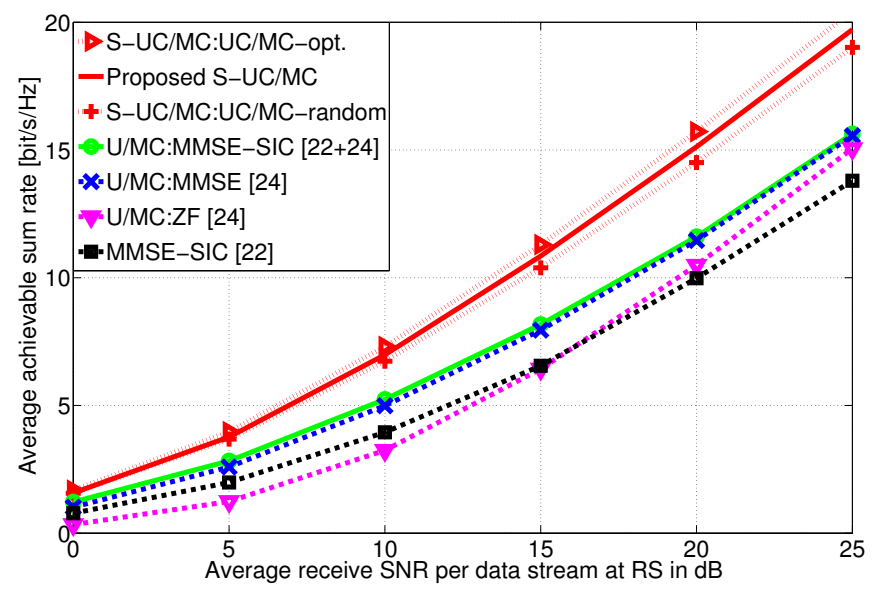

Fig. 4. Average achievable sum rates versus average receive SNR at RS, $G=1, N=4, L=4$.

transmit strategy utilizes the spatial processing capabilities at RS efficiently. U/MC:MMSE-SIC [22+24] performs similar to MMSE-SIC [22] for $L \leq 3$ and performs similar to U/MC:MMSE [24] for $L \geq 4$. Thus, the proposed S-UC/MC transmit strategy significantly outperforms a straightforward combination of spatial processing at RS and temporal processing at the nodes.

Fig. 4 shows the average achievable sum rates versus the average receive SNR at RS for the same scenario as above considering $L=4$ antennas at $\mathrm{RS}$. The approaches which are based on the proposed S-UC/MC transmit strategy outperform all other approaches over the entire SNR range. MMSE-SIC [22] benefits less from an increase of the average receive SNR at RS than the other approaches because random beamforming is considered at RS. The performance of U/MC:ZF [24] improves compared to the U/MC:MMSE [24] and MMSE-SIC [22] for increasing average SNRs because the impact of the noise enhancement due to the spatial separation of all signals at RS decreases.

Fig. 5 shows the average achievable sum rates versus the number of antennas at RS for a scenario consisting of $G=1$ group with $N=10$ single-antenna nodes.

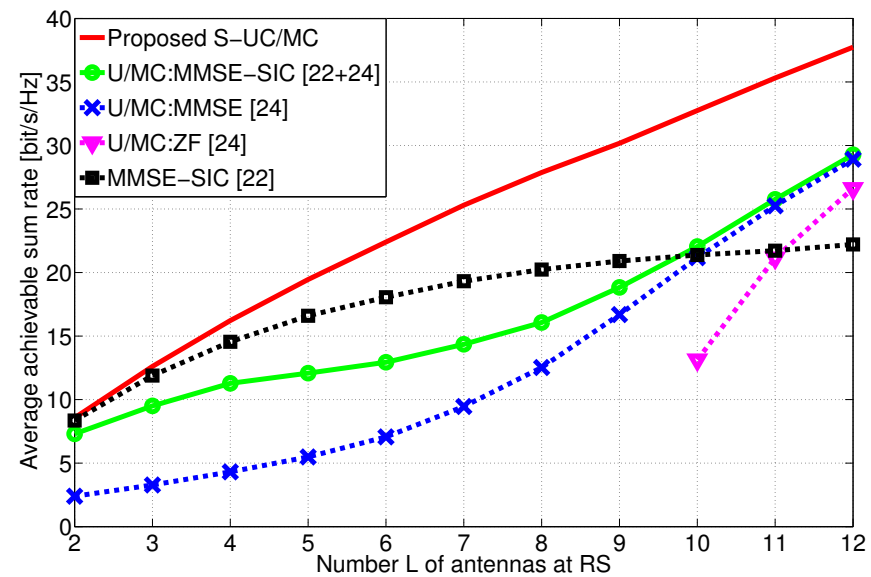

Fig. 5. Average achievable sum rates versus number $L$ of antennas at RS for an average receive $\mathrm{SNR}=15 \mathrm{~dB}$ at $\mathrm{RS}, G=1, N=10$.

For these simulations, we set the average receive SNR at $\mathrm{RS}$ to $15 \mathrm{~dB}$. U/MC:ZF [24] starts from $L=10$ antennas because $L \geq N$ antennas are required to spatially separate all signals. For $L=2$ antennas at RS, the proposed relay transceive filter design has no advantage compared to random beamforming because too many signals are simultaneously received at RS. Thus, MMSE-SIC [22] and the proposed S-UC/MC transmit strategy achieve similar average sum rates. For an increasing number of antennas at RS, the performance gain of the proposed S-UC/MC transmit strategy compared to MMSE-SIC [22] increases due to exploiting the spatial processing capabilities at RS, e.g., the gain is approximately $17 \%$ for $L=5$ and $53 \%$ for $L=10$. The performance gain of the proposed S-UC/MC transmit strategy compared to U/MC:MMSE [24] decreases for an increasing number of antennas at RS because the relative gain of efficiently exploiting temporal receive processing at the nodes decreases, e.g., the gain is approximately $81 \%$ for $L=9$ and $31 \%$ for $L=12$. U/MC:MMSE-SIC [22+24] achieves data rates in between MMSE-SIC [22] and U/MC:MMSE [24] for $L<10$ because the linear dependencies between the retransmitted signals are higher compared to MMSE-SIC [22]. For $L \geq 10$, U/MC:MMSE-SIC [22+24] performs similar to U/MC:MMSE [24]. The performance gain of the proposed S-UC/MC transmit strategy is significant compared to U/MC:MMSE-SIC [22+24] which is based on a straightforward combination of spatial and temporal processing.

\section{B. Multi-Group Multi-Way Scenario}

Fig. 6 and Fig. 7 show the average achievable sum rates versus the number of antennas at RS for a scenario consisting of $G=2$ groups with $N=3$ and $N=5$ single antenna nodes, respectively. For these simulations, the average receive SNR at RS is $15 \mathrm{~dB}$ and the approach of [22] is not considered because this approach does not enable a spatial separation of multiple groups. U/MC:ZF [24] requires $L \geq 6$ or $L \geq 10$ antennas at RS to spatially separate the received signals in case of $N=3$ or $N=5$ nodes per group, respectively. The proposed S-UC/MC transmit strategy outperforms all other 


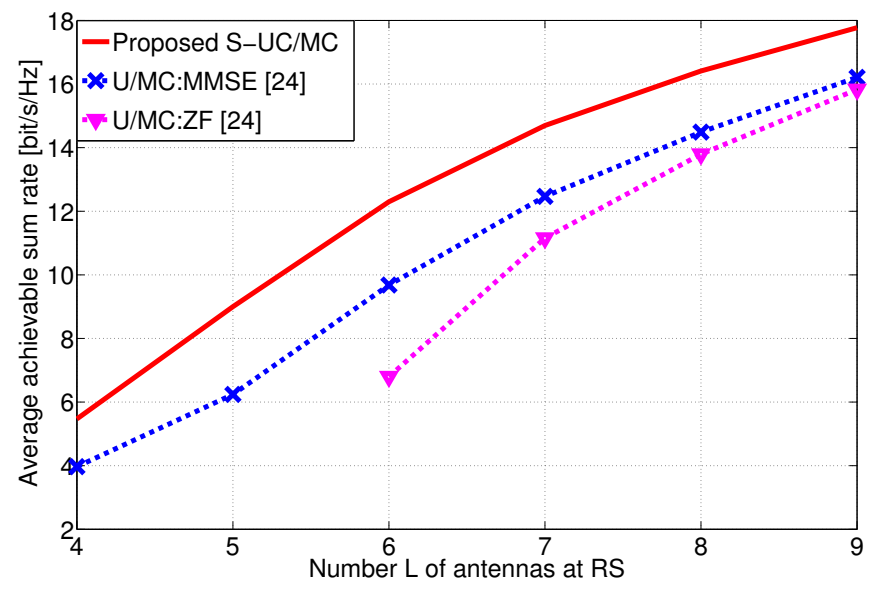

Fig. 6. Average achievable sum rates versus number $L$ of antennas at RS for an average receive $\mathrm{SNR}=15 \mathrm{~dB}$ at $\mathrm{RS}, G=2, N=3$.

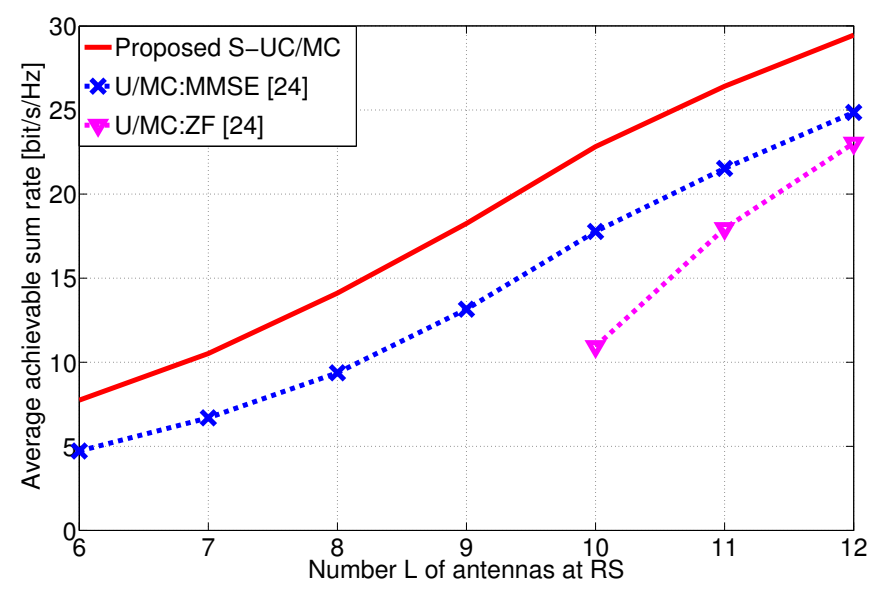

Fig. 7. Average achievable sum rates versus number $L$ of antennas at RS for an average receive $\mathrm{SNR}=15 \mathrm{~dB}$ at $\mathrm{RS}, G=2, N=5$.

approaches. In detail, the performance gain of the proposed S-UC/MC transmit strategy compared to U/MC:MMSE [24] decreases for increasing the number of antennas at RS, e.g., in case of $N=3$ the gain is approximately $44 \%$ for $L=5$ and $13 \%$ for $L=8$. The performance gain of the proposed $\mathrm{S}-\mathrm{UC} / \mathrm{MC}$ transmit strategy compared to U/MC:MMSE [24] increases for increasing the number $N$ of nodes per group because the temporal processing gain at the nodes increases due to increasing the number of $\mathrm{BC}$ phases, e.g., in case of $N=5$ the gain is approximately $51 \%$ for $L=8$.

\section{TDMA versus Spatial Separation}

Up to now, we have only considered spatial separation of multiple groups. However, orthogonal multiple access schemes like TDMA can also be used to separate the communications of multiple groups. Thus, we compare the performance of considering spatial separation with the performance of considering TDMA to separate multiple groups in the following. Fig. 8 shows the average achievable sum rates versus the number of antennas at RS for a scenario consisting of $G=2$ groups with $N=4$ single antenna nodes. For the simulations, the average receive $\mathrm{SNR}$ at $\mathrm{RS}$ is $15 \mathrm{~dB}$. The approach of

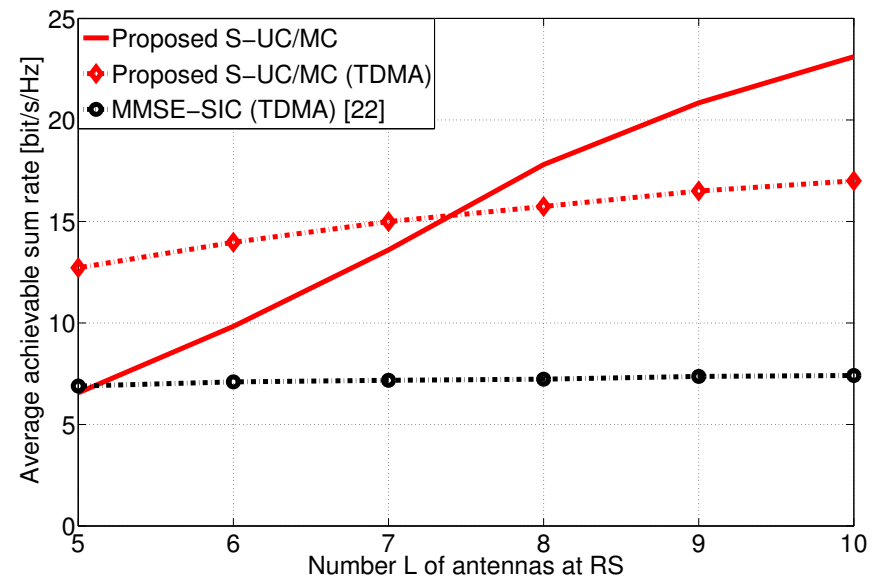

Fig. 8. Average achievable sum rates versus number $L$ of antennas at RS for an average receive $\mathrm{SNR}=15 \mathrm{~dB}$ at $\mathrm{RS}, G=2, N=4$.

[22] is considered using TDMA to separate the communications of the two groups, MMSE-SIC (TDMA) [22]. For our proposed S-UC/MC transmit strategy, we consider two different approaches to separate the communications of the two groups. First, we consider spatial separation of the groups using the proposed relay transceive filter design. Secondly, we consider TDMA to separate the communications of both groups. The spatial separation and the TDMA approach are termed S-UC/MC and S-UC/MC (TDMA), respectively. The S-UC/MC (TDMA) approach outperforms the other approaches for $L<K$. For $L>(G-1) N+2=N+2$, the proposed S-UC/MC transmit strategy with spatial separation outperforms MMSE-SIC (TDMA) [22] and for $L \geq K$ it performs better than the proposed S-UC/MC (TDMA) approach. To summarize, with $L<K$ antennas at RS, it is better to separate different groups in time to zero-force the inter-group interferences. For $L \geq K$ antennas at $\mathrm{RS}$, the groups can be well separated in space. Thus, a time division is not required and higher data rates can be achieved if the nodes transmit simultaneously.

\section{CONCLUSIONS}

We have proposed a superimposed uni-/multicasting transmit strategy for non-regenerative multi-group multi-way relaying which efficiently combines spatial transceive processing at RS and joint temporal receive processing at the nodes. To enable an efficient combination, we have proposed to exploit ANC. Thus, we have proposed to retransmit spatially processed linear combinations of all received signals in each BC phase such that the spatial processing capabilities at RS are utilized efficiently and the temporal processing capabilities at the nodes can be exploited to recover the individual signals. Furthermore, for the spatial processing at RS, we have derived an MMSE based closed-form solution for an ANC aware relay transceive filter. Additionally, we have presented lowcomplexity approaches to determine a UC/MC decoding order and to select the UC and the MC signals. By numerical results, we have shown that the proposed transmit strategy significantly outperforms existing approaches. 


\section{APPENDIX}

In this appendix, derivations for the relay transceive filter design of Section IV are presented. From the Lagrangian function (19), the Karush-Kuhn-Tucker (KKT) conditions can be derived:

$$
\begin{aligned}
\frac{\partial L}{\partial \mathbf{G}_{t}}=\sum_{l=1}^{K} \frac{\partial F\left(\mathbf{G}_{t}, \alpha_{t}, t, l\right)}{\partial \mathbf{G}_{t}}-\eta \mathbf{G}_{t}^{*} \mathbf{\Upsilon}^{\mathrm{T}} & =\mathbf{0} \\
\frac{\partial L}{\partial \alpha_{t}}=\sum_{l=1}^{K} \frac{\partial F\left(\mathbf{G}_{t}, \alpha_{t}, t, l\right)}{\partial \alpha_{t}} & =0, \\
\eta\left(\operatorname{tr}\left(\mathbf{G}_{t} \mathbf{\Upsilon} \mathbf{G}_{t}^{\mathrm{H}}\right)-P_{\mathrm{RS}}\right) & =0
\end{aligned}
$$

where

$$
\begin{aligned}
\frac{\partial F\left(\mathbf{G}_{t}, \alpha_{t}, t, l\right)}{\partial \mathbf{G}_{t}}= & -\alpha_{t} d_{l, t} \mathbf{h}_{l} \mathbf{h}_{k}^{\mathrm{T}} P_{M S} \\
& +\left|\alpha_{t}\right|^{2}\left|d_{l, t}\right|^{2} \mathbf{h}_{l} \mathbf{h}_{l}^{\mathrm{H}} \mathbf{G}_{t}^{*} \mathbf{\Upsilon}^{(k)^{\mathrm{T}}} \\
& +\left|\alpha_{t}\right|^{2}\left|d_{l, t}\right|^{2} \mathbf{h}_{l} \mathbf{h}_{l}^{\mathrm{H}} \mathbf{G}_{t}^{*} \mathbf{\Upsilon}^{\left(o_{t}\right)^{\mathrm{T}}} \\
& +\left|\alpha_{t}\right|^{2}\left|d_{l, t}\right|^{2} \mathbf{h}_{l} \mathbf{h}_{l}^{\mathrm{H}} \mathbf{G}_{t}^{*} \sigma_{n, \mathrm{RS}}^{2},
\end{aligned}
$$

and

$$
\begin{aligned}
\frac{\partial F\left(\mathbf{G}_{t}, \alpha_{t}, t, l\right)}{\partial \alpha_{t}}= & -d_{l, t} \mathbf{h}_{l}^{\mathrm{T}} \mathbf{G}_{t} \mathbf{h}_{k} P_{M S} \\
& +\alpha_{t}^{*}\left|d_{l, t}\right|^{2} \mathbf{h}_{l}^{\mathrm{T}} \mathbf{G}_{t} \mathbf{\Upsilon}^{(k)} \mathbf{G}_{t}^{\mathrm{H}} \mathbf{h}_{l}^{*} \\
& +\alpha_{t}^{*}\left|d_{l, t}\right|^{2} \mathbf{h}_{l}^{\mathrm{T}} \mathbf{G}_{t} \mathbf{\Upsilon}^{\left(o_{t}\right)} \mathbf{G}_{t}^{\mathrm{H}} \mathbf{h}_{l}^{*} \\
& +\alpha_{t}^{*}\left|d_{l, t}\right|^{2}\left(\mathbf{h}_{l}^{\mathrm{T}} \mathbf{G}_{t} \mathbf{G}_{t}^{\mathrm{H}} \mathbf{h}_{l}^{*} \sigma_{n, \mathrm{RS}}^{2}+\sigma_{n}^{2}\right) .
\end{aligned}
$$

Using the second KKT condition, we can write $\alpha_{t}^{*}$ as

$$
\alpha_{t}^{*}=\frac{\sum_{l=1}^{K} d_{l, t} \mathbf{h}_{l}^{\mathrm{T}} \mathbf{G}_{t} \mathbf{h}_{k} P_{M S}}{\sum_{l=1}^{K} b_{l, t}}
$$

where

$$
\begin{aligned}
b_{l, t}= & \left|d_{l, t}\right|^{2} \mathbf{h}_{l}^{\mathrm{T}} \mathbf{G}_{t} \mathbf{\Upsilon}^{(k)} \mathbf{G}_{t}^{\mathrm{H}} \mathbf{h}_{l}^{*}+\left|d_{l, t}\right|^{2} \mathbf{h}_{l}^{\mathrm{T}} \mathbf{G}_{t} \mathbf{\Upsilon}^{\left(o_{t}\right)} \mathbf{G}_{t}^{\mathrm{H}} \mathbf{h}_{l}^{*} \\
& +\left|d_{l, t}\right|^{2}\left(\mathbf{h}_{l}^{\mathrm{T}} \mathbf{G}_{t} \mathbf{G}_{t}^{\mathrm{H}} \mathbf{h}_{l}^{*} \sigma_{n, \mathrm{RS}}^{2}+\sigma_{n}^{2}\right)
\end{aligned}
$$

Now, $\alpha_{t}$ can be inserted in the first KKT condition (33a). Afterwards, the condition can be multiplied by $\mathbf{G}^{\mathrm{T}}$ and the trace operator can be applied. Furthermore, the transpose operation and some algebraic manipulations can be performed, yielding

$$
\eta\left(\operatorname{tr}\left(\mathbf{G}_{t} \Upsilon \mathbf{\Upsilon} \mathbf{G}_{t}^{\mathrm{H}}\right)=-\left|\alpha_{t}\right|^{2} \sigma_{n}^{2} \sum_{l=1}^{K}\left|d_{l, t}\right|^{2} .\right.
$$

Considering the predefined receive filters at the nodes with $\left|d_{l, t}\right|^{2}=1$ and using the third KKT condition (33c), we obtain

$$
\eta=-\frac{\left|\alpha_{t}\right|^{2} K \sigma_{n}^{2}}{P_{\mathrm{RS}}}
$$

\section{REFERENCES}

[1] D. Gunduz, A. Yener, A. Goldsmith, and H. Poor, "The multi-way relay channel," IEEE Transactions on Information Theory, vol. 59, no. 1, 2013.

[2] — , "The multi-way relay channel," in IEEE International Symposium on Information Theory, 2009, pp. 339-343.

[3] X. Tang and Y. Hua, "Optimal design of non-regenerative MIMO wireless relays," IEEE Transactions on Wireless Communications, vol. 6 , no. 4, pp. 1398-1407, 2007.

[4] O. Muñoz Medina, J. Vidal, and A. Agustín, "Linear transceiver design in nonregenerative relays with channel state information," IEEE Transactions on Signal Processing, vol. 55, no. 6, pp. 2593-2604, 2007.

[5] I. Hammerström and A. Wittneben, "Power allocation schemes for amplify-and-forward MIMO-OFDM relay links," IEEE Transactions on Wireless Communications, vol. 6, no. 8, pp. 2798-2802, 2007.

[6] Y. Rong, X. Tang, and Y. Hua, "A unified framework for optimizing linear nonregenerative multicarrier MIMO relay communication systems," IEEE Transactions on Signal Processing, vol. 57, no. 12, pp. 4837-4851, 2009.

[7] B. Rankov and A. Wittneben, "Spectral efficient protocols for halfduplex fading relay channels," IEEE Journal on Selected Areas in Communications, vol. 25, no. 2, pp. 379-389, 2007.

[8] T. Unger, "Multi-antenna two-hop relaying for bi-directional transmission in wireless communication systems," Ph.D. dissertation, TU Darmstadt, June 2009.

[9] R. Zhang, Y.-C. Liang, C. C. Chai, and S. Cui, "Optimal beamforming for two-way multi-antenna relay channel with analogue network coding," IEEE Journal on Selected Areas in Communications, vol. 27, no. 5, pp. 699-712, 2009.

[10] F. Roemer and M. Haardt, "Algebraic norm-maximizing (ANOMAX) transmit strategy for two-way relaying with MIMO amplify and forward relays," IEEE Signal Processing Letters, vol. 16, no. 10, pp. 909-912, 2009.

[11] S. Xu and Y. Hua, "Optimal design of spatial source-and-relay matrices for a non-regenerative two-way MIMO relay system," IEEE Transactions on Wireless Communications, vol. 10, no. 5, pp. 1645-1655, 2011.

[12] Y. Rong, "Joint source and relay optimization for two-way linear non-regenerative MIMO relay communications," IEEE Transactions on Signal Processing, vol. 60, no. 12, pp. 6533-6546, 2012.

[13] R. Wang and M. Tao, "Joint source and relay precoding designs for MIMO two-way relaying based on MSE criterion," IEEE Transactions on Signal Processing, vol. 60, no. 3, pp. 1352-1365, 2012.

[14] E. Yilmaz, R. Zakhour, D. Gesbert, and R. Knopp, "Multi-pair twoway relay channel with multiple antenna relay station," in Proc. IEEE International Conference on Communications (ICC), 2010, pp. 1-5.

[15] A. Amah and A. Klein, "Pair-aware transceive beamforming for nonregenerative multi-user two-way relaying," in Proc. IEEE International Conference on Acoustics Speech and Signal Processing (ICASSP), 2010, pp. 2506-2509.

[16] C. Y. Leow, Z. Ding, K. Leung, and D. Goeckel, "On the study of analogue network coding for multi-pair, bidirectional relay channels," IEEE Transactions on Wireless Communications, vol. 10, no. 2, pp. 670681, 2011.

[17] Z. Zhao, Z. Ding, M. Peng, W. Wang, and K. Leung, "A special case of multi-way relay channel: When beamforming is not applicable," IEEE Transactions on Wireless Communications, vol. 10, no. 7, pp. 20462051, 2011.

[18] M. Tao and R. Wang, "Linear precoding for multi-pair two-way MIMO relay systems with max-min fairness," IEEE Transactions on Signal Processing, vol. 60, no. 10, pp. 5361-5370, 2012.

[19] J. Joung and A. Sayed, "Multiuser two-way amplify-and-forward relay processing and power control methods for beamforming systems," IEEE Transactions on Signal Processing, vol. 58, no. 3, pp. 1833-1846, 2010.

[20] H. Degenhardt and A. Klein, "Self-interference aware MIMO filter design for non-regenerative multi-pair two-way relaying," in Proc. IEEE Wireless Communications and Networking Conference (WCNC), 2012.

[21] T. Cui, T. Ho, and J. Kliewer, "Space-time communication protocols for n-way relay networks," in IEEE Global Telecommunications Conference, 2008, pp. 1-5.

[22] J. Cao and Z. Zhong, "Non-regenerative multi-way relaying: Ordered MMSE-SIC receivers exploiting temporal diversity," in IEEE 75th Vehicular Technology Conference (VTC Spring), 2012, pp. 1-5.

[23] A. Amah and A. Klein, "Beamforming-based physical layer network coding for non-regenerative multi-way relaying," EURASIP Journal on Wireless Communications and Networking, Special Issue on Physical Layer Network Coding for Wireless Cooperative Networks, 2010. 
[24] _ - "Non-regenerative multi-antenna multi-group multi-way relaying," EURASIP Journal on Wireless Communications and Networking, 2011.

[25] L. Ong, S. Johnson, and C. Kellett, "An optimal coding strategy for the binary multi-way relay channel," IEEE Communications Letters, vol. 14, no. 4 , pp. 330-332, 2010.

[26] _ _ "The capacity region of multiway relay channels over finite fields with full data exchange," IEEE Transactions on Information Theory, vol. 57, no. 5, pp. 3016-3031, 2011.

[27] L. Ong, C. M. Kellett, and S. J. Johnson, "On the equal-rate capacity of the awgn multiway relay channel," IEEE Transactions on Information Theory, vol. 58, no. 9, pp. 5761-5769, 2012.

[28] C. Hausl, O. Iscan, and F. Rossetto, "Resource allocation for asymmetric multi-way relay communication over orthogonal channels," EURASIP Journal on Wireless Communications and Networking, vol. 2012, no. 1, p. 20, 2012.

[29] A. Amah and A. Klein, "Regenerative multi-group multi-way relaying," IEEE Transactions on Vehicular Technology, vol. 60, no. 7, pp. 3017 3029, 2011.

[30] R. Ahlswede, N. Cai, S.-Y. Li, and R. Yeung, "Network information flow," IEEE Transactions on Information Theory, vol. 46, no. 4, pp. 1204-1216, 2000.

[31] S. Katti, S. Gollakota, and D. Katabi, "Embracing wireless interference: Analog network coding," in ACM SIGCOMM, 2007, pp. 397-408.

[32] B. Hassibi and B. Hochwald, "How much training is needed in multipleantenna wireless links?" IEEE Transactions on Information Theory, vol. 49, no. 4, pp. 951-963, 2003.

[33] F. Gao, R. Zhang, and Y.-C. Liang, "Optimal channel estimation and training design for two-way relay networks," IEEE Transactions on Communications, vol. 57, no. 10, pp. 3024-3033, 2009.

[34] B. Jiang, F. Gao, X. Gao, and A. Nallanathan, "Channel estimation and training design for two-way relay networks with power allocation," IEEE Transactions on Wireless Communications, vol. 9, no. 6, pp. 2022-2032, 2010.

[35] G. Wang, F. Gao, W. Chen, and C. Tellambura, "Channel estimation and training design for two-way relay networks in time-selective fading environments," IEEE Transactions on Wireless Communications, vol. 10 , no. 8, pp. 2681-2691, 2011.

[36] H. Degenhardt, F. Hohmann, and A. Klein, "Pilot transmission scheme and robust filter design for non-regenerative multi-pair two-way relaying," in Proc. of the IEEE International Symposium on Personal, Indoor and Mobile Radio Communications (PIMRC'12), 2012.

[37] M. Joham, "Optimization of linear and nonlinear transmit signal processing," Ph.D. dissertation, TU München, June 2004.

[38] M. Joham, W. Utschick, and J. Nossek, "Linear transmit processing in MIMO communications systems," IEEE Transactions on Signal Processing, vol. 53, no. 8, pp. 2700-2712, 2005.

[39] K. B. Petersen and M. S. Pedersen, The Matrix Cookbook. Technical University of Denmark, 2012.

[40] D. Tse and P. Viswanath, Fundamentals of Wireless Communication. Cambridge, U.K.: Cambridge Univ. Press, 2005.

[41] S. Boyd and L. Vandenberghe, Convex Optimization. Cambridge, U.K.: Cambridge Univ. Press, 2004. 